\title{
The PADME scientific Program
}

\author{
Paola Gianotti $^{a, *}$ for the PADME collaboration ${ }^{1}$ \\ ${ }^{a}$ Laboratori Nazionali di Frascati of INFN, \\ Via E. Fermi 54, I-00044 Frascati, Italy \\ E-mail: paola.gianotti@lnf.infn.it
}

The elusiveness of dark matter has triggered innovative and open-minded approaches involving also experiments at accelerators spanning a wide range of energies with high-sensitivity detectors. In spite of the variety of attempts, up-to-now none of the conducted experiments has produced any firm evidence. In this scenario is inserted the Positron Annihilation into Dark Matter Experiment (PADME) ongoing at the Frascati National Laboratory of INFN. PADME is searching a Dark Photon signal by studying the missing mass spectrum of single-photon final states resulting from the annihilation of positrons on the electrons of a fix target. After commissioning and beamline optimization, PADME collected in 2020 about $5 \times 10^{12}$ positrons on target. Actually, the PADME approach allows to look for any new light, feebly interacting particle produced in $e^{+} e^{-}$ annihilations through a virtual off-shell photon. The scientific program of the experiment, and its current status, are here illustrated.

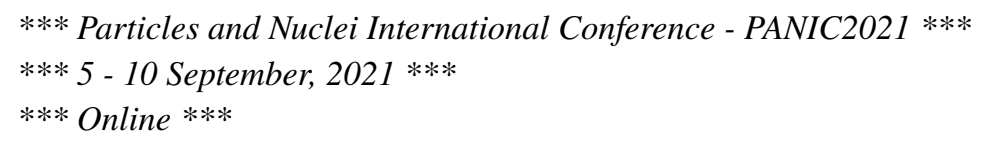

${ }^{1}$ A.P. Caricato, M. Martino, I. Oceano, F. Oliva, S. Spagnolo (INFN Lecce and Dip. di Matematica e Fisica, Università del Salento), G. Chiodini (INFN Lecce), F. Bossi, B. Buonomo, R. De Sangro, C. Di Giulio, D. Domenici, G. Finocchiaro, L.G. Foggetta, M. Garattini, A. Ghigo, F. Giacchino, P. Gianotti, I. Sarra, B. Sciascia, T. Spadaro, E. Spiriti, C. Taruggi, E. Vilucchi (INFN Laboratori Nazionali di Frascati), V. Kozhuharov (University of Sofia "St. Kl. Ohridski" and INFN Laboratori Nazionali di Frascati), G. Georgiev, S. Ivanov, R. Simeonov (University of Sofia “St. Kl. Ohridski”), F. Ferrarotto, E. Leonardi, F. Safai Tehrani, P. Valente (INFN Roma1), E. Long, G.C. Organtini, G. Piperno, M. Raggi (INFN Roma1 and Dip. di Fisica, "Sapienza" Università di Roma), S. Fiore (ENEA centro ricerche Frascati and INFN Roma1), V. Capirossi, F. Iazzi, F. Pinna (Politecnico di Torino), B. Liberti (Dip. di Fisica, Università di Roma "Tor Vergata", INFN Laboratori Nazionali di Frascati), M. Martini Università G. Marconi and INFN Laboratori Nazionali di Frascati), J. Alexander, A. Frankenthal (Dep. of Physics Cornell University)

*Speaker 


\section{Introduction}

One of the arguments motivating the search for extensions of the Standard Model (SM) is the need to explain the nature of Dark Matter (DM). The majority of the theoretical and experimental efforts conducted so far have been dedicated to test the hypothesis that DM corresponds to Weakly Interacting Massive Particles (WIMPs). However, despite extensive searches, up to now no conclusive signals of WIMPs have been identified [1]. This stimulated the scientific community to explore other hypotheses.

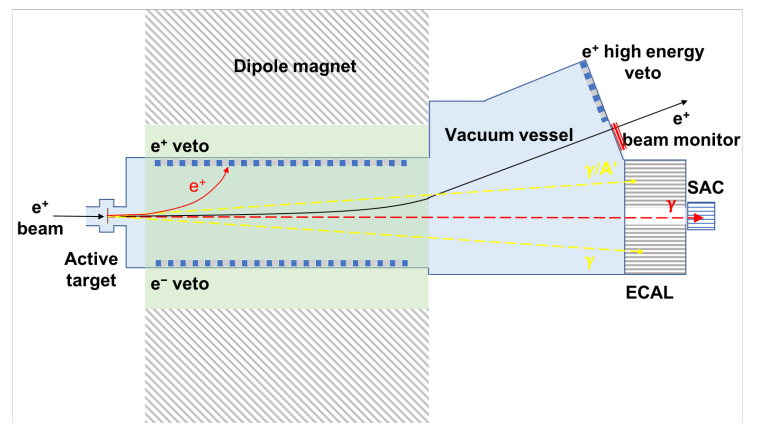

Figure 1: The layout of the PADME experiment. The main elements of the setup are pointed out. Tracks corresponding to different kind of events (Bremsstrahlung, $2 \gamma s$, etc.) are also shown.

An interesting alternative to the WIMP scenario is represented by Feebly Interacting Particles (FIPs) with mass below the electroweak symmetry-breaking scale whose interactions with SM fields are strongly suppressed [2]. FIPs are often assumed to be part of a possibly secluded sector, called the Dark Sector (DS), with the lightest stable dark particle(s) being responsible for the DM [3]. From the theoretical point of view, this alternative is well motivated [2] and could also help to explain some anomalies observed experimentally (i.e. muon $g-2$ value, proton charge radius, lepton-flavour universality violation etc.). FIPs scenario spans over a wide range of possibilities, depending from the nature of the new particles (scalars, pseudoscalars, fermions, spin-one bosons) and covers several order of magnitude in mass and couplings.

\section{Search for FIPs with the PADME experiment}

At the Frascati National Laboratory (LNF) of INFN, The Positron Annihilation into Dark Matter Experiment (PADME) is trying to explore some of the possibilities coming out form the FIPs approach [4]. The primary goal of PADME is to investigate a minimal vector-portal scenario for the DS hypothesis, by searching a massive $A^{\prime}$ Dark Photon (DP) corresponding to the gauge field of a new $U_{D}(1)$ symmetry responsible for interactions between dark matter particles. The mass, $m_{A^{\prime}}$, and the coupling $\epsilon$ to the SM particles, parametrized with a kinetic mixing term between the ordinary photon and the $A^{\prime}$, are the only two parameters necessary to describe this phenomenology. The $m_{A^{\prime}}$ value and the mass hierarchy of the DS, also define $A^{\prime}$ decay modes: if in the Dark Sector exist lighter particles that the DP, its dominant decay modes will be invisible; if otherwise the $A^{\prime}$ is the lighter particle of the DS, it could decay visibly in SM lepton pairs. 
The PADME detector has been designed to study the reaction $e^{+} e^{-} \rightarrow \gamma A^{\prime}$, with the $A^{\prime}$ decaying invisibly. This is realized by means of a positron beam, provided by the LINAC of the DAФNE complex [5, 6], annihilating with the electrons of a fix target. A sketch of the PADME detector is shown in fig. 1 (for a more detailed description of the apparatus see [7]).

The DP presence is determined by studying the missing mass of single-photon final states. With a maximum value of the positron beam energy of $550 \mathrm{MeV}, m_{A^{\prime}}$ values up to $23.7 \mathrm{MeV} / c^{2}$ can be explored. A sensitivity on the coupling parameter $\epsilon^{2} \geq 10^{-7}$ can be reached by collecting $\mathrm{O}\left(10^{13}\right)$ Positrons On Target (POT).

Actually, the range of physics subjects that the PADME detector can explore is wider and comprises other topics than the DP study. Some other peculiar final states are accessible via the $e^{+} e^{-}$annihilation and can be investigated with the present setup. Studies have been performed to determine the experiment sensitivity to the production of Axion-Like Particles (ALPs) or a Dark Higgs (DH). An Axion-Like Particle ( $a$ ) is another possible mediator between the SM and the DS. With pseudo-scalar quantum numbers and spin-0, it would decay $a \rightarrow e^{+} e^{-}$or in $2 \gamma s$ if no other dark sector particle lighter than the ALP exists [8]. The search for ALPs can proceed in parallel to that of the DP, if no hypotheses are made on its decays and a signal is only searched through the missing mass study of single-photon final states. Figure 2 shows the expected PADME sensitivities for DP and ALPs compared with that of other similar experiments.
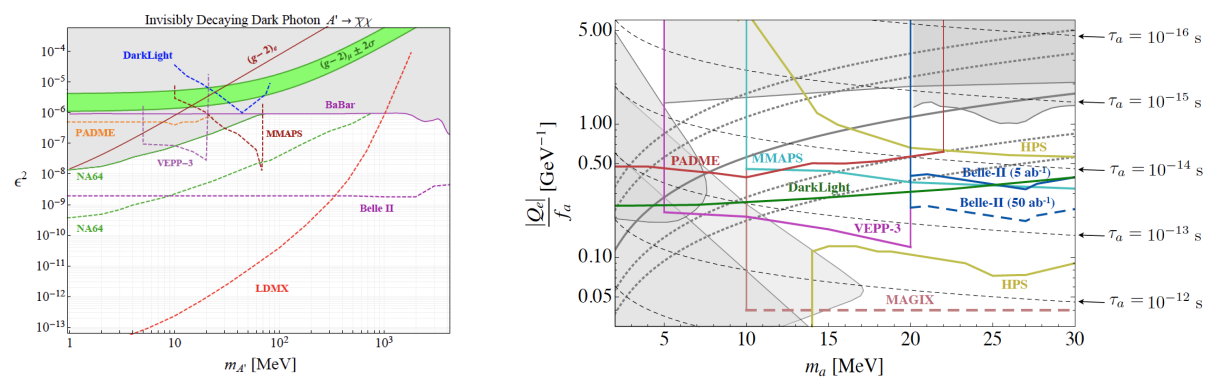

Figure 2: Left: current constraints (shaded regions) and sensitivity estimates (dashed lines) on the coupling of the $A^{\prime}$ for various experiments based on the missing mass, missing energy and missing momentum. The green band shows the values required to explain the muon $g-2$ anomaly (from [12]). Right: sensitivity to the ALPs coupling of ongoing and proposed experiments studying $e^{+} e^{-}$annihilations. Grey areas indicate excluded regions by past experiments. In the picture the curves illustrating $m_{a}$ vs $\left|Q_{e}\right| / f_{a}$ relation for different values of $\left|Q_{e}\right|$ are shown together with different values of ALPs lifetime $\tau_{a}$ (see [13] for more details).

Different is instead the situation for a Dark Higgs $\left(h^{\prime}\right)$. In a non-minimal model where the mass of the $A^{\prime}$ is the result of a spontaneous symmetry breaking, it is possible an associate production of a new boson, referred as Dark Higgs [14]. In this framework naturalness requires that the two particles have similar mass. The production of the $h^{\prime}$ can be realized via the so-called Higgs'strahlung mechanism: $e^{+} e^{-} \rightarrow h^{\prime} A^{\prime}$, while concerning its decays if the $h^{\prime}$ would be heavy, it will decay in two vectors $A^{\prime}$. Therefore, in the hypothesis that the $A^{\prime}$ decays into a lepton pair, the final state will count six leptons. On the contrary, if the $h^{\prime}$ would be light, it will decay via loop processes to leptons and/or hadrons. In this case the Dark Higgs would be a long-lived particle and will be mostly undetected [9]. The PADME experiment has at present a low efficiency in reconstructing 
Dark Higgs events due to the high number of leptons in the final state. To increase the detection efficiency for these low energy particles, one possibility could be to reduce the intensity of the magnetic field increasing the detection efficiency of the charged particles. Therefore to study DH production dedicated data takings will be necessary.

The last topic that the PADME experiment is planning to address regards the existence of the so called $X 17$ boson. This is a hypothetical new particle, whose presence has been proposed to explain an anomaly observed in nuclear de-excitaions of ${ }^{8} \mathrm{Be}$ [10] and ${ }^{4} \mathrm{He}$ [11]. At present, the only evidence of this new state is represented by the bumps observed in the angular distribution of $\left(e^{+}, e^{-}\right)$pairs resulting form internal production of excited nuclei. The PADME experiment has the unique opportunity, by properly tuning the positron beam energy, to excite the $X 17$ production via the $\mathrm{O}(\alpha)$ resonant annihilation process $e^{+} e^{-} \rightarrow X$. To perform such a measurement some changes in the detector setup would be necessary and a dedicated data taking will be carried out in 2022 .

\section{PADME status and prospects}

The PADME detector was commissioned between the end of 2018 and the beginning of 2019 . This first data taking, whose main goal was to calibrate the detector components, revealed an unexpected high level of background. This was caused by particles coming out from the positron production target able to reach the PADME detector, and by the positrons interacting with a $\mathrm{Be}$ window that separated the accelerator and the experiment vacuum. Thus, 2019 was entirely devoted to change the beam line and to study the best beam condition to realize the experiment.

In 2020, despite of the pandemic, the experiment took $\sim 5 \times 10^{12}$ POT that constitute the first fraction of data to address the PADME scientific program.
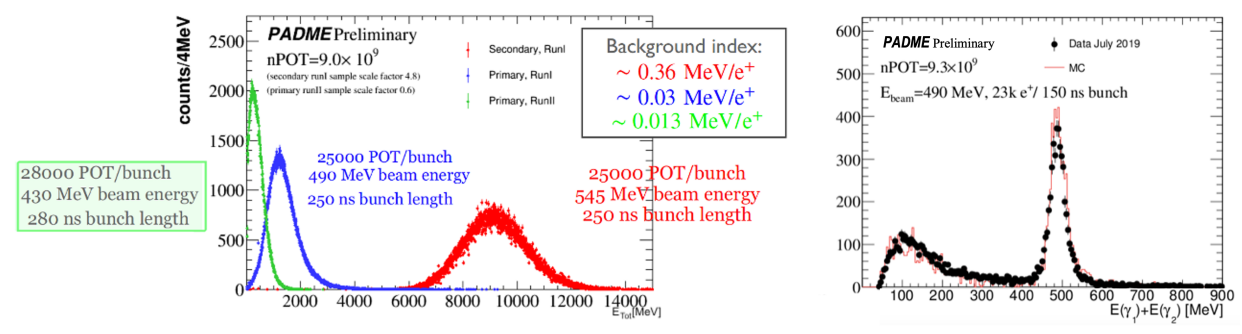

Figure 3: Left: total energy reconstructed by PADME e.m. calorimeter, without target. The colours refer to different run conditions highlighted in the figure. Thanks to an accurate beam line optimization, the background was reduced from 0.36 to $0.013 \mathrm{MeV} / e^{+}$. Right: Energy sum corresponding to the $e^{+} e^{-} \rightarrow \gamma \gamma$ final state. Black dots are data points, the red histogram represents MC expectation.

Before starting to address the search for FiPs, the PADME detector capabilities have to be tuned with known SM processes. In particular, the performance of the electromagnetic calorimeter to detect ordinary photons is of paramount importance. Figure 3-Left shows the total energy measured by e.m. calorimeter in special runs, without target, to study the beam-induced background. Starting form an initial energy of $\sim 0.36 \mathrm{GeV} / e^{+}$measured by the calorimeter at the end of 2018 , with proper changes in the positron production and some improvements of the whole beam line, the background was lowered down to $\sim 0.013 \mathrm{GeV} / e^{+}$. At present, the analysis efforts of the collaboration are 
direct to measure the $e^{+} e^{-} \rightarrow \gamma \gamma$ cross-section. On fig. 3-Right, it is shown the energy sum of two-photons events selected for this purpose. The evaluation of the 2-photon cross section is well advanced and a paper is in preparation.

\section{Conclusions}

The PADME experiment has a wide program to explore FIPs existence. This is done exploiting the possibilities offered by the low energy positron beam of the Frascati INFN laboratory. During the experiment commissioning, the detector demonstrated that the design performance has been reached and even overcame. The next steps for the collaboration are to analize the collected data to measure SM e.m. processes, as 2-photons annihilation cross-section, to fine tune all parameters and be ready to explore the Dark Sector.

\section{References}

[1] G. Arcadi et al., Eur. Phys. J. C 78 (3) (2018) 203. arXiv:1703.07364, doi:10.1140/epjc/s10052-018-5662-y.

[2] P. Agrawal et al., arXiv:2102.12143 [hep-ph].

[3] A. Celentano, L. Darmé, L. Marsicano and E. Nardi, Phys. Rev. D 102 (2020) no.7, 075026 doi:10.1103/PhysRevD.102.075026

[4] M. Raggi, V. Kozuharov, Adv. High En. Phys. 2014 (2014) 959802.

[5] G. Vignola et al., first $\Phi$-factory Conf Proc C960610 (1996) 22-26.

[6] P. Valente, B. Buonomo, C. Di Giulio and L. G. Foggetta, Proceedings of IBIC2016, Barcelona, Spain, doi:10.18429/JACoW-IBIC2016-MOPG65.

[7] F. Pinna for the PADME collaboration, this proceedings.

[8] L. Darmè, F. Giacchino, E. Nardi, M. Raggi, arXiv:2012.07894 [hep-ph].

[9] P. Ciafaloni, G. Martelli and M. Raggi, JHEP 04 (2021) 163.

[10] A. J. Krasznahorkay et al., Phys. Rev. Lett. textbf116 (2016), no. 4042501

[11] A. J. Krasznahorkay et al., Phys. Rev. C 104 (2021) no.4, 044003

[12] M. Battaglieri et al., Dark Matter 2017: Community Report, arXiv:1707.04591v1.

[13] D. S. M. Alves and N. Weiner, JHEP 07 (2018), 092

[14] B. Batell, M. Pospelov and A. Ritz, Phys. Rev. D 79 (2009) 115008. doi:10.1103/PhysRevD.79.115008

[15] P. Albicocco, J. Alexander, F. Bossi, P. Branchini, B. Buonomo, C. Capoccia, E. Capitolo, G. Chiodini, A. P. Caricato and R. de Sangro, et al. JINST 15 (2020) no.10, T10003 